\title{
The Automatic Radio Transmission Systems Recognition
}

\author{
Leszek Nowosielski, Bogdan Uljasz \\ Faculty of Electronics, Military University of Technology, \\ 2, S. Kaliskiego St. 00-908, Warsaw, Poland \\ leszek.nowosielski@wat.edu.pl
}

\begin{abstract}
This article provides a detailed description of methods for automatic recognition of transmission protocols, used in radio channels, based on distinctive characteristics of the radio signal received as well as binary sequence existing at the demodulator output of the radio recognition receiver. Conclusions are presented as relates to the operational efficiency of the signal analysis methods described, followed by possibilities to use them in practice. A software application has been presented, making use of methods for automatic transmission protocols recognition which are described in this article.
\end{abstract}

Index Terms-Telecommunications; radio channels; protocols recognition; coefficient of correlation; binary patterns.

\section{INTRODUCTION}

At present, specified military as well as civil applications within the radio recognition area make use of equipment that automatically recognises, demodulates as well as decodes signals sent by radio transmission systems, which use system-specific transmission protocols. From the point of view of the operator of equipment for automatic recognition and decoding radio transmission protocols it is important that characteristic parameters for a given protocol are recognised automatically. After the data transmission system applied has been recognized appropriately, the process of decoding information transferred should start pursuant to the protocol-specific rules.

Because of trade secrets of companies who offer equipment for automatic recognition of radio transmission systems for sale on the market, there is no precise description of algorithms for the recognition of radio transmission protocols available in the professional literature [1], [2]. Technical descriptions of recognition systems available on the market include only technical parameters and operational capabilities of the above systems. This article provides a detailed description of methods for automatic recognition of transmission protocols, used by the Authors in radio channels, based on distinctive characteristics of the radio signal received as well as binary sequence occurring at the demodulator output of the radio recognition receiver. Conclusions are presented as relates to

Manuscript received 5 May, 2017; accepted 14 October, 2017. the operational efficiency of the signal analysis methods described were presented, followed by possibilities to use them in practice. Currently, Universal Digital Demodulator (UDD) is being developed at the Telecommunications Institute of Military University of Technology in Poland. In its final version the UDD is going to automatically recognize, demodulate and decode radio transmission signals with specific system rules for the recognized transmission. A Universal Digital Decoder block diagram is presented in Fig. 1. The block responsible for carrying out the transmission protocol procedure which is described in this article is marked with green colour in Fig. 1.

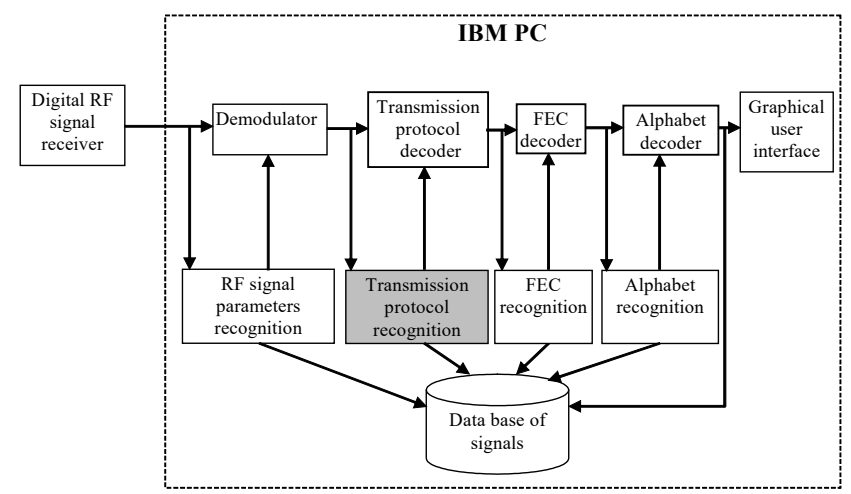

Fig. 1. Universal Digital Decoder block diagram.

A recognition algorithm proposed for radio transmission systems consists of three steps (Fig. 2):

- modulation recognition,

- technical parameters analysing binary sequence at the demodulator output,

- classification of binary sequence.

In case of some radio signals under analysis it may be necessary to apply the above mentioned algorithm repeatedly, because there is no possibility for unequivocal decision to be made as to the modulation type, e.g. QPSK and DQPSK. In this case one should apply a principle based on the concept of neighbourhood which makes use of certain defined similarity measures for various types of modulations, followed by analysis using also adjacent execution possibilities.

In the most modern solutions that use super-computers, parallel processing is applied which enables verifying simultaneously several possible realisations of the 
transmission system being recognised. It ensures unequivocal recognition result to be achieved faster. Each path executing such processing consists of functional blocks. While looking for a solution on can use heuristic algorithms with the method of discrete optimisation of bundle scanning (Fig. 3).

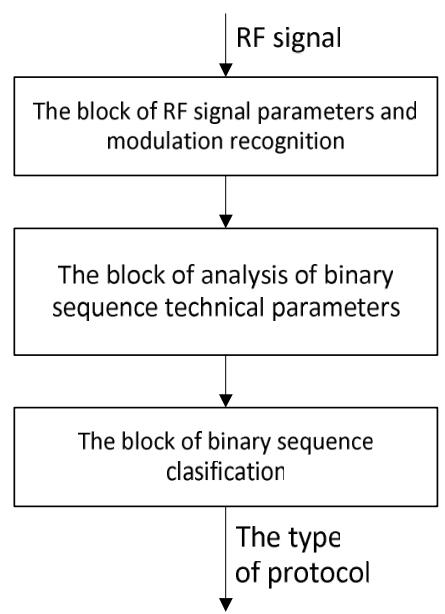

Fig. 2. Stages of the process of automatic radio transmission system recognition.

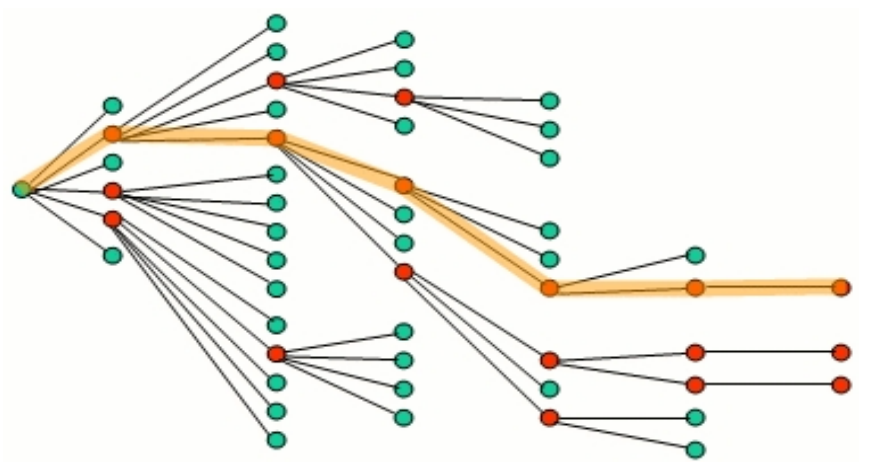

Fig. 3. Bundle scanning [3].

Each step of the analysis can offer many solutions, which are considered independently in order to achieve the most reliable solution in relation to the goal to be achieved, i.e. radio transmission system recognition. It is also possible to combine two methods, one for recognising basic characteristics of the signal, and the other for seeking detailed parameters. Ultimately it is important that the transmission system recognition is as much reliable as possible. During the recognition process it is necessary to minimise error probability in decision making. Scanning according to the highest probability principle is one of the well-known methods of minimising error probability in the decision making process, provided that all solutions are equally probable. This principle is applied in the Viterbi algorithm [4], which is generally used to decode convolution codes. The Viterbi algorithm can be used and implemented successfully in algorithms for recognition of parameters relating to radio transmission systems.

The Viterbi algorithm is used for finding a sequence of parameters specific for a given transmission system $\vec{s}(D)$, which features the highest conditional probability

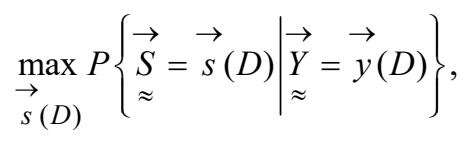

that the random variable $\underset{\approx}{\vec{S}}$ representing sequences of parameters specific for all possible transmission systems used will assume the value of $\vec{s}(D)$ provided that the received sequence $\vec{y}(D)$ is known.

By applying the Bayes theorem one can demonstrate that this task can be reduced to searching for a sequence of parameters specific for a given transmission system $\vec{s}(D)$ which maximises the conditional probability

$$
\max _{\vec{s}(D)} P\left\{\begin{array}{l}
\vec{Y}=\vec{y}(D) \\
\approx
\end{array} \mid \underset{\approx}{\vec{S}}=\vec{s}(D)\right\},
$$

of a given sequence of parameters $\vec{y}(D)$ when a parameter sequence $\vec{s}(D)$ is fixed.

The process of modulation recognition has not been described in this article. Only the two consecutive processes occurring after the modulation recognition process have been discussed:

- analysis of technical parameters of a binary sequence at the demodulator output,

- classification of the binary sequence.

When discussing algorithms of signal analysis in the two subsequent processes only those parameters were used, which can be obtained as a result of modulation recognition. In this article the Authors have focused on discussing the process of analysing technical parameters of a binary sequence at the demodulator output as well as on the binary sequence classification process. During these processes it is important that the received binary sequence is automatically classified at the demodulator output. After appropriate recognition of the data transmission system used, the recognition equipment should start decoding the information transferred, according to principles specific for the transmission system recognised.

The process of automatic recognition of transmission protocols is possible thanks to the existence of parameters which are specific for a given transmission system. Further in this article those parameters are called distinctive characteristics, specific of particular radio transmission systems. Below there is a list of distinctive characteristics of radio signals, which make it possible to recognise data transmission system automatically, followed by a description of methods of analysis thereof. The description includes methods based on calculating the correlation coefficient between autocorrelation of a binary sequence received and a pattern sequence as well as methods used for filtering the presence of binary patterns. 


\section{DistinCTIVE CHARACTERISTICS OF RADIO SigNALS Which ENABLE AUTOMATIC RECOGNITION}

A variety of signal processing methods (transmission coding, redundant coding, interleaving, scrambling, etc.) in one transmission system as well as effects of the signal processing methods overlapping make the recognition process very complicated. For example, even if a binary interleave type used were successfully recognized, phasing the interleaving process with the use of redundant coding in the next stage would be problematic.

More realistic approach to the recognition problem is an attempt at assigning the emission received - based on its distinctive characteristics - to one of the standard transmission systems (described in the literature [4]-[12]). Each transmission system features certain defined and system-specific transmission parameters. Those parameters include i.a.:

- Carrier frequency;

- Type of modulation;

- Modulation parameters (e.g. frequency shifting for FSK emission);

- Mode of operation (synchronous, asynchronous);

- Alphabet;

- Type of redundant coding;

- Type of scrambler used;

- Length of stop bit for asynchronous transmission;

- Characteristics of a given transmission protocol: headers, addresses, control sequences, synchronous sequences, padding sequences;

- Autocorrelation function of the binary sequence representative of a given radio transmission system.

Some of those parameters are the same for many data transmission systems. Due to this reason, while making a decision on the radio transmission system type, one must not rely only on the knowledge about a value of one of the parameters mentioned above. The decision making process should take account of the biggest possible set of parameters.

In order to speed up the recognition process it is worth trying to group systems according to common distinctive characteristics. This will make it possible to reduce the number of known transmission protocols to be analysed in the consecutive steps of the recognition process, resulting in a smaller set subject to analysis. Parameters presented can be used as steps of a bundle searching algorithm.

Effective recognition of radio transmission systems while making use of methods described further below is conditional upon the development of a database of radio transmission systems which would include the systemspecific parameters (distinctive characteristics) mentioned above. In this way a set $X$ of finite states will be defined for the purposes of achieving the goal of the recognition process.

\section{Automatic ReCOGNITION OF TRANSMISSION PROTOCOLS}

This chapter offers a description of methods of classifying binary sequence which occurs at the demodulator output. A binary sequence technical parameters analysis block is the element which precedes the classification arrangement. Data achieved from the binary sequence technical parameters analysis block as well as data achieved from the modulation recognition process done earlier is used for making the final decision on assignment of a given signal to a given type of the radio transmission system. The location of binary sequence arrangement within the classification system of radio signals is presented in Fig. 1.

Data delivered by the binary sequence technical parameters analysis arrangement include:

- Result of a test of hypothesis with zero correlation coefficient between the bit autocorrelation function of the binary sequence analysed and standard bit autocorrelation functions of binary sequences stored in the database, complying with particular transmission systems;

- Result of a test for the occurrence of binary patterns typical for particular transmission systems in the binary sequence analysed.

Within the binary sequence classification block [13]-[14], based on the parameters delivered by the modulation recognition arrangement, initial selection is done of radio transmission systems from among all types of radio transmission systems stored in the database. Also, the final assignment of the binary sequence received to a defined radio data transmission system is done within the binary sequence classification block, based on parameters delivered by the binary sequence technical parameters analysis arrangement. In case of incompatibility between the parameters delivered by the modulation recognition arrangement and those delivered by the binary sequence technical parameters analysis arrangement with the relevant parameters describing particular transmission systems which are recorded in the database, the equipment operator will be informed about non-recognition of the transmission system received. The operator can also be informed that analysis was carried out based on one of the possible modulations or coding methods (this process can also be carried out automatically by processing many modulation variants simultaneously).

An appropriately designed database of technical parameters for transmission systems currently used in radio communications (a set of states) is a necessary element in the correct operation of the binary sequence classification block. Table I presents an exemplary format of a database which enables ordering information about the selected transmission systems.

Particular columns of Table I include parameters delivered by the modulation recognition arrangement as well as achieved from earlier analysis of representative binary sequences assigned to transmission systems considered:

- column 1 includes the name of transmission system,

- columns 2 and 3 include the beginning and the end of the frequency range where a given transmission system is used most frequently,

- column 4 includes modulation type used by a given transmission system,

- column 5 includes modulation parameters (for FSK 
modulation the parameter includes frequency shift, for ASK, PSK and QAM modulations this field shall be empty),

- column 6 includes rate of modulation,

- column 7 includes mode of operation, two variants being possible here (synchronous or asynchronous),

- column 8 includes a name of the file with a stored autocorrelation function of standard binary sequence for a given transmission system (this function is used during the test of hypothesis with zero correlation coefficient between the bit autocorrelation function of the binary sequence analysed and standard bit autocorrelation function of binary sequence for the system with the name entered in column 1),

- column 9 includes identification of the alphabet used in a given transmission system,

- column 10 includes a threshold value of the frequency of defined binary standards specific for a given transmission system.

This data is used for achieving the goal, namely system recognition, by means of applying the greedy algorithm (the final decision is built step-by-step). In case of multi-plot processing it is possible to use the bundle searching algorithm providing possibility to verify many solutions simultaneously. In the variant of analysing one bundle only, in case of failure, the analysis will not be started from the beginning; it will start from the nearest lower node with lower probability from the recognition.

Data included in columns $1 \div 7$ is used during initial selection of the transmission system based on data achieved from the modulation parameters recognition arrangement. Here, the elimination method is applied.

Data included in column 8 is used during radio transmission system selection based on the binary sequence analysis while applying the test of hypothesis with zero correlation coefficient between the bit autocorrelation function of the binary sequence analysed and standard bit autocorrelation function of binary sequence described in the database.

Data stored in column 10 is used during transmission system selection based on the binary sequence analysis while applying the test of occurrence of the binary standard typical of the transmission system described in the database.

Data stored in column 9 is used during alphabet decoding.

A. Selection of Transmission Systems Based on Parameters Delivered by Modulation Recognition Arrangement

Because of standardisation used in relation to types of data transmission systems and also of unique values of some technical parameters of information transporting signals, many transmission systems used can be unequivocally identified based on technical parameters of signals delivered by the modulation recognition arrangement.

Thanks to the knowledge of particular values of given technical parameters it is possible to select such systems from the database, the modulation parameters of which are compatible with the parameters of the signal received from the transmission system. A rate of modulation used is a quite distinctive parameter in relation to protocols. Analysis of this parameter can be troublesome in many cases, especially in the presence of degrading noise components. Therefore, it is necessary to define acceptable error of modulation rate measurement made by the modulation recognition arrangement. Some transmission systems use modulation rates which are considerably different from the typical commonly used series of types, e.g. $49.5 \mathrm{Bd}$ [10]. This is also the case with the FSK modulation.

During the process of comparing process, delivered by the modulation recognition arrangement with the parameters corresponding to transmission systems stored in the database, the following events may occur:

- conformity of the parameters compared. In this case a given transmission system will be qualified for further analysis,

- nonconformity of the parameters compared (at least in case of one parameter), in this case a given transmission system will not be qualified for further analysis,

- the value of a given parameter is not recorded in the database (because the value is unknown). In this case during the comparison process the parameter, the value of which is not stored in the database is ignored and the decision about qualification of the given transmission system for further analysis is made according to the conformity or non-conformity of the remaining parameters.

TABLE I. EXEMPLARY FORMAT OF RADIO TRANSMISSION SYSTEM PARAMETERS DATABASE.

\begin{tabular}{|c|c|c|c|c|c|c|c|c|c|}
\hline $\begin{array}{c}\text { Transmission } \\
\text { system name }\end{array}$ & $\begin{array}{c}\text { Beginning of } \\
\text { frequency } \\
\text { range } \\
{[\mathbf{M H z}]}\end{array}$ & $\begin{array}{c}\text { End of } \\
\text { frequency } \\
\text { range } \\
{[\mathbf{M H z}]}\end{array}$ & $\begin{array}{c}\text { Type of } \\
\text { modulation }\end{array}$ & $\begin{array}{c}\text { Frequency } \\
\text { shift }\end{array}$ & $\begin{array}{c}\text { Rate of } \\
\text { modulation } \\
{[\mathbf{B d}]}\end{array}$ & $\begin{array}{c}\text { Transmission } \\
\text { mode }\end{array}$ & $\begin{array}{c}\text { Autocorrelation } \\
\text { standard }\end{array}$ & $\begin{array}{c}\text { Threshold } \\
\text { Aalphabet } \\
\text { vorrelation } \\
\text { coefficient }\end{array}$ \\
\hline $\mathbf{1}$ & $\mathbf{2}$ & $\mathbf{3}$ & $\mathbf{4}$ & $\mathbf{5}$ & $\mathbf{6}$ & $\mathbf{7}$ & $\mathbf{8}$ & $\mathbf{9}$ & $\mathbf{1 0}$ \\
\hline RTTY_5 & 3 & 30 & FSK & 850 & 0 & asynch & 0 & 0 & 0.8 \\
\hline RTTY_5 & 3 & 300 & FSK & 425 & 0 & asynch & 0 & 0 & 0.8 \\
\hline RTTY_5 & 30 & 300 & FSK & 170 & 0 & asynch & 0 & 0 & 0.8 \\
\hline RTTY_7 & 3 & 30 & FSK & 425 & 0 & asynch & 0 & 0 & 0.8 \\
\hline RTTY_7 & 30 & 300 & FSK & 170 & 0 & asynch & 0 & 0 & 0.8 \\
\hline AMTOR_FEC & 30 & 300 & FSK & 170 & 100 & synch & 0 & CCITT_476 & 0.3 \\
\hline AMTOR_ARQ & 30 & 300 & FSK & 170 & 100 & synch & Aut_Amtor_arq.txt & CCITT_476 & 0.7 \\
\hline MIL_STD_188_- & 1 & 30 & 8PSK & 0 & 2400 & synch & 0 & 0 & 0.7 \\
\hline 110A & 3 & 300 & ASK & 0 & 0 & asynch & 0 & MORS & 0.7 \\
\hline MORS & 3 & 30 & 8FSK & 250 & 125 & synch & 0 & ASCII_8 & 0.7 \\
\hline MIL_STD_188_ & $341 A$ & 30 & FSK & 250 & 300 & synch & 0 & 0 & 0.7 \\
\hline AX_25 & 3 & & &
\end{tabular}




\section{B. Selecting Data Transmission System Based on Data} Delivered by the Binary Sequence Technical Parameters Analysis Arrangement from the Demodulator Output

An analysis of the demodulated binary sequence may make it possible to correctly identify an unknown data transmission protocol. Specific characteristics of the binary sequence structure corresponding to a defined transmission system (i.e. number of bits describing a single character, length of frame, repetition cycle, synchronising sequences) can be easily described by the estimate of bit autocorrelation function of the binary sequence occurring at the demodulator output. A specific nature of the autocorrelation function enables using it as a signature of a given type of data transmission system.

Further in the article there is a description of the test of hypothesis with zero correlation coefficient between the bit autocorrelation function of the binary sequence analysed and standard bit autocorrelation functions of binary sequences stored in the database, which correspond to particular radio transmission systems. As a result of the above mentioned test it will be possible to easily assign a binary sequence analysed to a defined transmission system.

Another method of assigning a given binary sequence to the defined data transmission system is looking for distinctive patterns within binary data sequences characteristic for this system only. The results of the above mentioned test as well as the results of the test with zero correlation coefficient between the bit autocorrelation function of the binary sequence analysed and standard bit autocorrelation functions of binary sequences stored in the database will allow for unequivocal assigning of the binary sequence analysed to the defined transmission system.

During the decision making process carried out by the classification block of the binary sequence the following events may occur:

- the results of both tests related to a defined transmission system are positive (in this case the similarity of binary sequence analysed to the transmission system it was compared with scores 2 points),

- the results of both tests related to a defined transmission system are negative (in this case similarity of binary sequence analysed to the transmission system it was compared with scores 0 points),

- the results of both tests related to a defined transmission system are different (in this case similarity of binary sequence analysed to the transmission system it was compared with scores 1 point).

Finally, the binary sequence analysed is assigned to the transmission system which scored 2 points. Is several transmission systems score 2 points each, then the decision about assigning the binary sequence analysed to a defined system is up to the operator. If none of the transmission systems stored in the database scores 2 points, then the operator will be informed which of the systems scored 1 point. Systems which scored 0 points will not be analysed further.

1) Test of the Hypothesis with Zero Correlation Coefficient

The existence of relations between two random variables and their intensities can be measured by expressing them by means of a correlation coefficient $\rho$. In a simple case of two random variables $x$ and $y$ the correlation coefficient is expressed by an equation

$$
\rho_{x y}=\frac{C_{x y}}{\rho_{x} \rho_{y}},
$$

where $C_{x y}$ is the covariance of the variables $x$ and $y$.

Assuming that the random variables $x$ and $y$ are sampled, then $N$ pairs of values is achieved. Thus, the correlation coefficient can then be estimated based on this data as follows

$$
r_{x y}=\hat{\rho}_{x y}=\frac{\sum_{i=1}^{N}\left(x_{i}-\bar{x}\right)\left(y_{i}-\bar{y}\right)}{\left[\sum_{i=1}^{N}\left(x_{i}-\bar{x}\right)^{2} \sum_{i=1}^{N}\left(y_{i}-\bar{y}\right)^{2}\right]^{1 / 2}} .
$$

Similar to $\rho_{x y}$, the correlation coefficient of the $r_{x y}$ sample is included in the range from -1 to +1 and achieves the limiting values only when the observation results show a strictly linear dependence. Both non-linear dependence and dispersion of the results make the $r_{x y}$ value of approach zero.

By means of determining estimators of the bit autocorrelation function of binary sequences for particular transmission systems one may develop a database of signatures (in the form of shape of particular autocorrelation function), based on which classification will be carried out. A correlation coefficient can be used as a similarity measure between autocorrelation of a binary sequence occurring at the demodulator output and standard autocorrelation functions of binary sequences corresponding to particular transmission systems stored in the database.

By means of determining values of correlation coefficients between autocorrelation of the binary sequence occurring at the demodulator output and all standard autocorrelation functions of the binary sequences corresponding to particular transmission systems stored in the database it is possible, while using the principle of the smallest distance to select a pattern which is the most similar to the autocorrelation function of the binary sequence analysed, and as a result to determine its affiliation. The standard transmission system, which achieves the highest value of the correlation coefficient is deemed as corresponding to the binary sequence received. Such a solution has a disadvantage in that even in case of very small correlation coefficient values (autocorrelation functions analysed are hardly similar) this method will always give some result, which in many cases may lead to improper classification of the binary sequence under analysis.

Another method of making a decision on assigning a binary sequence under analysis to a defined data transmission system is to carry out the hypothesis test with zero correlation coefficient between the bit autocorrelation function of the binary sequence analysed and particular standard autocorrelation functions stored in the database. 
It is based on verifying whether non-zero correlation coefficient value really reflects the existence of considerable statistical correlation between autocorrelation functions we are interested in. Such a checkup is based on verifying the hypothesis that $r_{x y}=0$. If the hypothesis must be rejected, it means that considerable correlation exists.

When determining the acceptance range of the hypothesis with zero correlation coefficient it is advisable to use a special function of the $r_{x y}$ argument, namely

$$
w=\frac{1}{2} \ln \frac{1+r_{x y}}{1-r_{x y}} .
$$

The distribution of the random variable $w$ is approximately normal, and the average value and variance are expressed by means of the following formula:

$$
\begin{gathered}
\mu_{w}=\frac{1}{2} \ln \frac{1+r_{x y}}{1-r_{x y}}, \\
\sigma_{w}^{2}=\frac{1}{N-3} .
\end{gathered}
$$

As a result of equations under (6), the distribution of the variable $w$ sample at $r_{x y}=0$ is normal with the average value of $\mu_{w}=0$ and variance $\sigma_{w}^{2}=\frac{1}{N-3}$. Therefore, the range of acceptance for the hypothesis with the zero correlation coefficient is as follows

$$
-z_{\alpha / 2}<=\frac{\sqrt{N-3}}{2} \ln \frac{1+r_{x y}}{1-r_{x y}}<z_{\alpha / 2},
$$

where $z$ is a standardised random variable

$$
z=\frac{x-\mu_{x}}{\sigma_{x}},
$$

with normal distribution. The values located out of the above mentioned range show that there exists a statistical correlation at the level of confidence $\alpha$.

When carrying out the test of hypothesis with zero correlation coefficient it should be verified that the correlation coefficient calculated for particular pattern autocorrelation functions and autocorrelation function for the binary sequence analysed satisfies inequality (8). Before starting the calculations, a defined value of the confidence level $\alpha$ of the test should be assumed. For ascending values of the parameter $\alpha$ the acceptance range of the test will be going down.

\section{2) Test of Occurrence of Binary Patterns Typical for Transmission System}

Most of transmission protocols can be characterised by a form of protocol-specific binary pattern which occurs at the demodulator output. This characteristic enables recognising a given protocol automatically. Below, a description of the analysing algorithm for a binary sequence is presented using the test of occurrence of the binary pattern typical for the transmission system described in the database. The above algorithm involves searching binary sequences specific for a given protocol of the binary sequence received in the binary stream of length $n$ and calculating the conformity rate $K-$ which is defined as the ratio of number of binary vectors occurring in the received binary vectors sequence of defined length, consistent with the pattern, in relation to the number of all binary vectors with a given length, contained in the bit stream received. If the calculated conformity rate exceeds a defined threshold stored in the database, then the decision is made as to conformity of the binary sequence received with a defined transmission system. Figure 4 presents a simplified algorithm for recognising the transmission system described above.

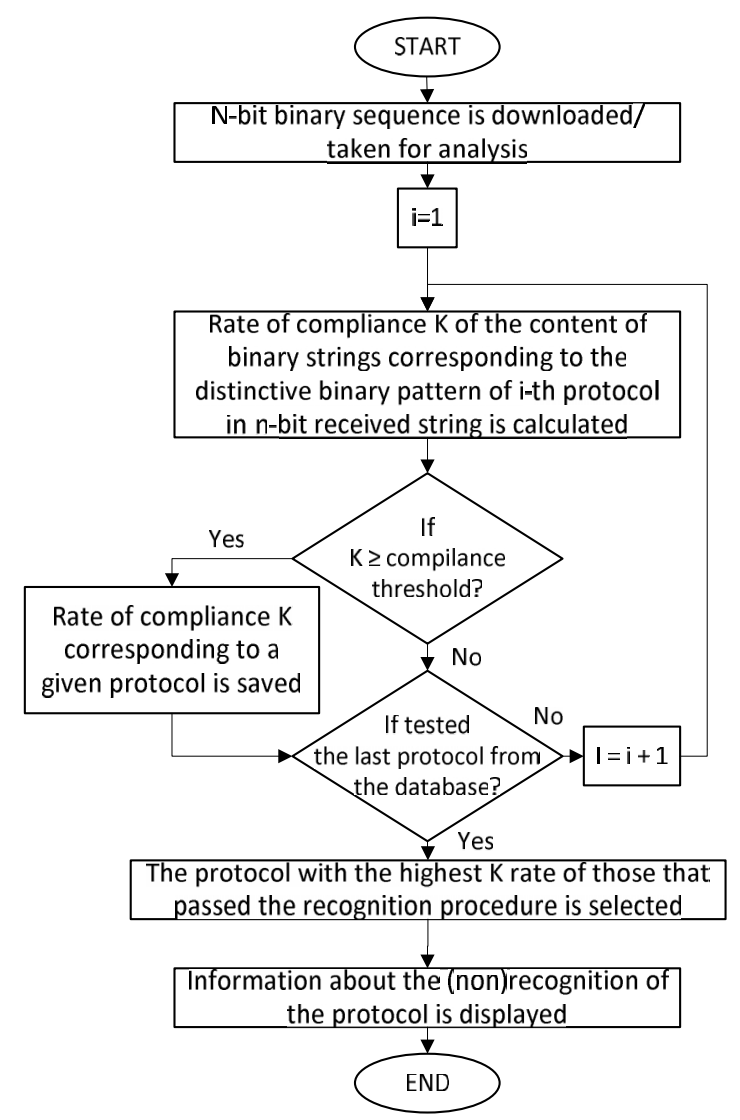

Fig. 4. Algorithm of the transmission protocols recognising procedure.

In order to test the operation effectiveness of the procedure based on the algorithm proposed, a series computer simulations were carried out where the probability estimate of the detection correctness $P_{w}$ was measured for selected radio transmission systems as the function of error occurrence probability $p$ in the BSC (Binary Symmetric Channel) channel. The tests were carried out for simple transmission systems, both synchronous and asynchronous ones. The results of simulations for transmission systems such as: MORS, RTTY-5, AMTOR FEC, AMTOR ARQ, FELD HELD and PSK 31 are presented in the Fig. 5. Based on the simulation results achieved it can be stated that the method proposed can be successfully used in practical applications to be used for automatic recognition of transmission systems. Such good results achieved for simple transmission systems allow also to infer about good results for more complicates systems. 


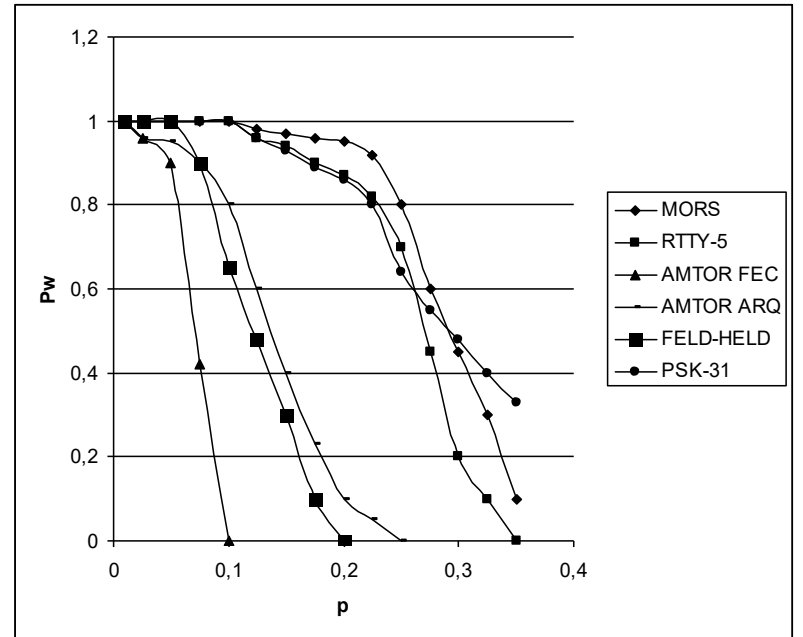

Fig. 5. Probabilities of correct detection $P_{w}$ of selected transmission systems as the $\mathrm{w}$ function of the error $p$ occurrence probability in the BSC channel.

\section{COMPUTER APPLICATION}

Within the framework of one of projects executed by the Authors of this article the algorithms for recognising radio transmission systems described above have been implemented in the form of PC computer applications executing the recognition process. The main window of the application is presented in the Fig. 6. On the right side of the figure there are areas related to recognising basic radio signal parameters. These areas are related with the initial recognising parameters necessary for the recognition of radio protocols, redundant codes and alphabets.

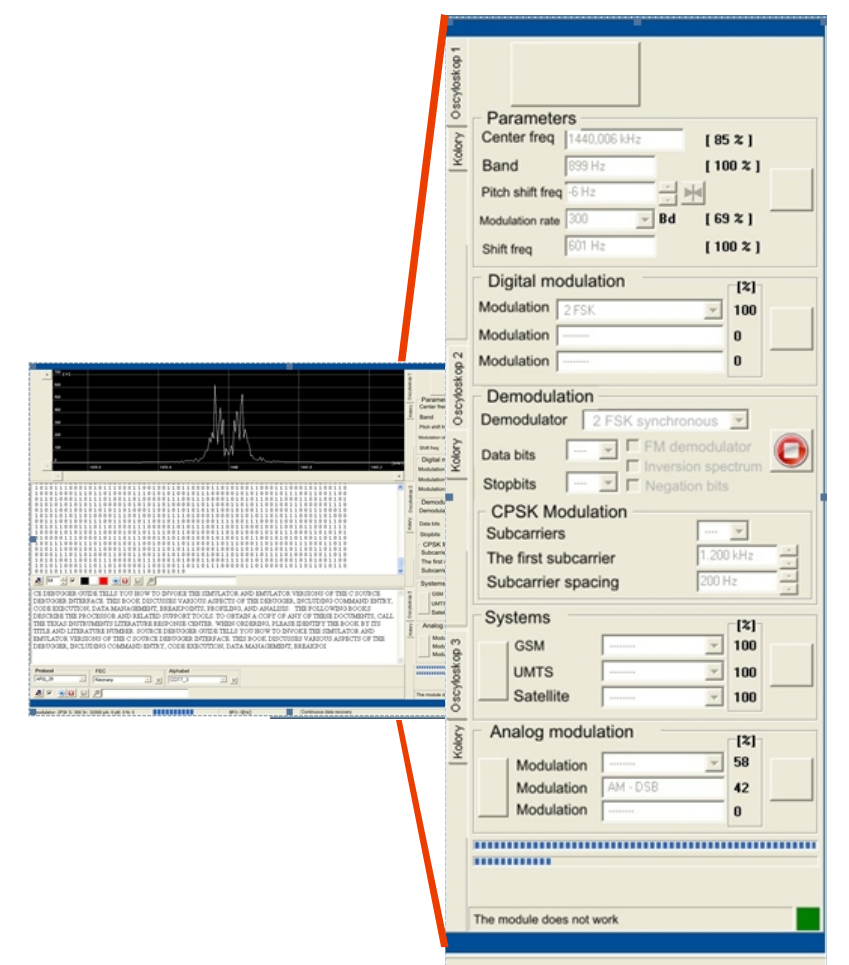

Fig. 6. A view of the program window includes the decoded text together with transmission parameters.

Figure 7 presents two program windows. The first window presents the values of demodulated bits - the results of demodulator operation. The second window shows the contents of message decoded after the recognition of radio protocol, redundant coding FEC and the alphabet.

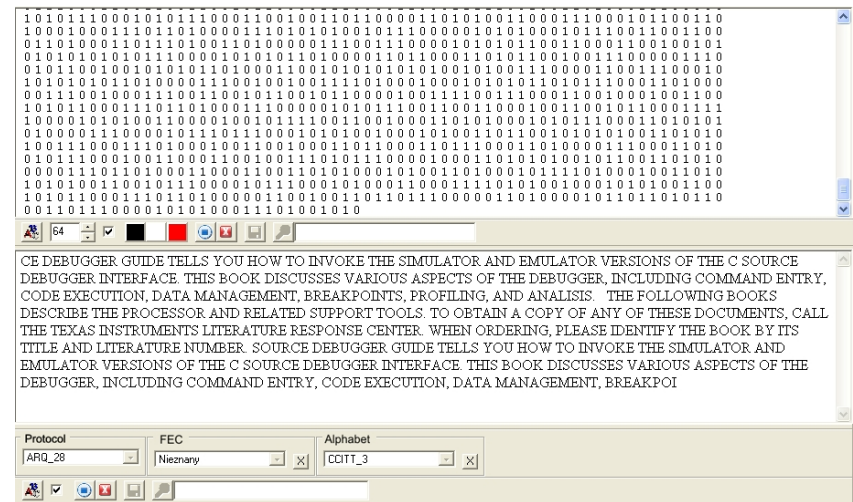

Fig. 7. A view of windows with values of demodulated bits and decoded text of the message received.

Protocols, redundant codes FEC as well as alphabets are recognised automatically by matching suitable solutions from the pool of applications types known and introduced to the memory. The application enables adding new protocols, redundant codes and alphabets using a simple text editor. The algorithms of applications used for automatic recognising of FEC redundant codes are described in the literature item [15].

\section{CONCLUSIONS}

This article in details proprietary methods of automatic recognition of transmission protocols used in radio channels, which methods are based on distinctive characteristics of the received radio signal as well as binary sequence occurring at the output of the radio recognition receiver demodulator.

A description was presented of a software application dedicated to the recognition process, which was developed under one of the projects carried out by the Authors hereof. The application described makes use of radio transmission systems recognition algorithms.

The results of computer simulation were presented, during which the operating efficiency of the recognition procedure for transmission systems was measured, based on the method of filtering the occurrence of binary patterns typical for a defined transmission system. Based on the simulation results achieved it can be stated that the method proposed ensures good effects for errors generated in a channel using a model of the discrete channel BSC.

\section{REFERENCES}

[1] J. Kingenfuss, Radio data code manual. Kingenfuss publications.

[2] Manual, FSK Analyzer GA 082, Rohde\&Schwarz, Germany.

[3] „Problemy optymalizacji dyskretnej. Algorytmy heurystyczne”, Polsko-Japońska Akademia Technik Komputerowych, [Online]. Available: http://edu.pjwstk.edu.pl/wyklady/nai/scb/wyklad5/w5.htm (in Polish).

[4] Shu Lin, D. J. Costello, Error control coding, fundamentals and applications. Prentice-Hall, 2007.

[5] H. Liu, R. Liao, Z. Wei, Z. Hou, Y. Qiao, "BER Analysis of a hybrid modulation scheme based on PPM and MSK subcarrier intensity modulation", IEEE Photonics Journal, vol. 7, pp. 720-1510, 2015. [Online]. Available: http://dx.doi.org/10.1109/JPHOT.2015.2449265

[6] K. Dabrowski, Amatorska komunikacja cyfrowa. Wydawnictwo naukowe PWN, Poland, Warszawa, 1994. (in Polish).

[7] L. Komsta, Poradnik - krótkofalarstwo i radiokomunikacja. Wydawnictwa Komunikacji i Lacznosci, Poland, Warszawa, 2001. (in Polish). 
[8] ITU-R M. 476-5 Recommendation: Direct-printing telegraph equipment in the maritime mobile service, 2001, ITU Article Number E8403. [Online]. Available: https://www.itu.int/rec/R-REC-M.476-5199510-I/en

[9] ITU-R M. 625-3 Recommendation: Direct-printing telegraph equipment employing automatic identification in the maritime mobile service, 2001, ITU Article Number E8490. [Online]. Available: https://www.itu.int/rec/R-REC-M.625-3-199510-S/en

[10] R. Proesch, Technical Handbook for Radio Monitoring HF. Books on Demand GmbH, Norderstedt, Germany, 2015.

[11] R. Proesch, A. Daskalaki-Proesch, Technical Handbook for Radio Monitoring VHF/UHF. Books on Demand $\mathrm{GmbH}$, Norderstedt, Germany, 2015.

[12] R. Proesch, Signal Analyzis for Radio Monitoring. Books on Demand
GmbH, Norderstedt, Germany, 2015.

[13] R. Dinuls, G. Erins, A. Lorencs, I. Mednieks, J. Sinica-Sinavskis, "Tree species identification in mixed Baltic forest using LiDAR and multispectral data", IEEE Selected Topics Appl. Earth Observ. Remote Sensing, vol. 5, no. 2, pp. 594-603, 2012. [Online]. Available: http://dx.doi.org/10.1109/JSTARS.2012.2196978

[14] S. Sun, H. Shi, Y. Wu, "A survey of multi-source domain adaptation", Information Fusion, vol. 24, pp. 84-92, 2015. [Online]. Available: http://dx.doi.org/10.1016/j.inffus.2014.12.003

[15] L. Nowosielski, M. Wnuk, "The performance of the algorithm for automatic FEC codes recognition", 20th Int. Conf. Microwaves, Radar and Wireless Communications, (MIKON 2014), Poland, 2014 [Online]. Available: http://dx.doi.org/10.1109/MIKON.2014. 